\title{
A Review on Novel Drug Targets and Future Directions for COVID-19 Treatment
}

This article was published in the following Dove Press journal:

Biologics: Targets and Therapy

\author{
Yohannes Tsegyie Wondmkun' \\ Ousman Ahmed Mohammed (iD ${ }^{2}$ \\ 'Department of Pharmacology and \\ Clinical Pharmacy, School of Pharmacy, \\ College of Health Sciences, Addis Ababa \\ University, Addis Ababa, Ethiopia; \\ ${ }^{2}$ College of Health Sciences, Woldia \\ University, Woldia, Ethiopia
}

\begin{abstract}
Severe acute respiratory syndrome coronavirus-2 causes coronavirus disease-19 (COVID-19) that spreads quickly in the world. Considering the impact of this pandemic, researchers have been racing to understand the peculiar nature of the virus and the pathogenesis of the disease to uncover possible drug targets, effective therapeutic agents, and vaccines. Accordingly, numerous drug targets are identified by scientists. Among them, structural glycoproteins, virulence factors, host-specific receptors and enzymes, nonstructure proteins, the Janus kinase/signal transducers and activators of transcription (JAK/ STAT) signaling pathway, and pro-inflammatory cytokines are discussed herein. This review summarizes the promising drug targets for COVID-19, and highlights antiviral strategies which depend on molecular interactions between viral small molecules and host biologic machinery for repurposing the available clinical drugs. In addition, it gives a strong rational basis for the ongoing discovery of new drugs and vaccines.
\end{abstract}

Keywords: COVID-19, drug targets, SARS-COV-2, non-structural proteins, structural proteins

\section{Introduction}

The coronavirus disease-2019 (COVID-19) pandemic due to the emergence of severe acute respiratory syndrome coronavirus-2 (SARS-COV-2) sustains to spread swiftly across the globe. As of July 26, 2020, more than 16.2 million confirmed cases were reported from 213 countries. ${ }^{1}$ To date, deaths have surpassed 648,866. ${ }^{1}$ Currently, some clinical studies reported the use of a few drugs in severe cases. Of these, the steroid dexamethasone improves survival in severe cases of COVID-19. ${ }^{2}$ In addition, another study revealed that compassionate use of remdesivir produced clinical improvement in $68 \%$ of hospitalized patients with severe cases of COVID-19, though measurement of efficacy requires ongoing randomized, placebo controlled trials. ${ }^{3}$ In the face of all this burden, researchers were looking for the identification of potential drug targets to SARS-COV-2. Most recent findings give strong attention to proteins which are involved in viral nucleic acid synthesis and replication, structural proteins that have a role in viral entry and fusion, virulence factors, host-specific receptors and enzymes, pro-inflammatory cytokines, and immune response modulating signaling pathways. ${ }^{4-9}$ Therefore, the following sections dwell on the aforementioned targets, physiological features, structural makeup and some pipeline antiviral drugs.

\section{The Viral RNA Synthesis and Replication}

Non-structural proteins (Nsps), functional proteins, participate in viral replication and infection of the host by inducing transcription and translation of viral RNA.

\footnotetext{
Correspondence: Yohannes Tsegyie Wondmkun

Department of Pharmacology and Clinical Pharmacy, School of Pharmacy, College of Health Sciences, Addis Ababa University, Churchill Avenue, P.O. Box II76, Addis Ababa, Ethiopia

Email yohannes.tsegyie@aau.edu.et
}

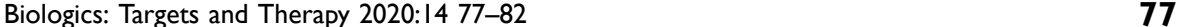

(c) (i) (c) 2020 Wondmkun and Mohammed. This work is published and licensed by Dove Medical Press Limited. The full terms of this license are available at https://www. BY dovepress.com/terms.php and incorporate the Creative Commons Attribution - Non Commercial (unported, v3.0) License (http://creativecommons.org/licenses/by-nc/3.0/). By accessing the work you hereby accept the Terms. Non-commercial uses of the work are permitted without any further permission from Dove Medical Press Limited, provided the work is properly attributed. For permission for commercial use of this work, please see paragraphs 4.2 and 5 of our Terms (https://www.dovepress.com/terms.php). 
These proteins are getting the current and future attention as drug targets for COVID-19 treatment. Among them, 3-chymotrypsin-like protease (3CLpro), RNA-dependent RNA polymerase (RdRp), papain-like protease (PLpro), and helicase are the most considered targets for the discovery of different therapeutic agents due to their clear vital enzyme active site and biological functions. ${ }^{4}$

\section{RNA-Dependent RNA Polymerase (RdRp)}

RdRp also known as Nsp12 is a conserved protein in COVID-19 which is an essential enzyme for RNA transcription and replication of this virus. The RdRp domain of polymerase is located at the C-terminus, and has a conserved Ser-Asp-Asp motif. ${ }^{4}$ Enzymatic activity and binding of Nsp12 to RNA is increased by the Nsp7-Nsp8 complex. ${ }^{5,6}$ On the other hand, inhibition of RdRp is one of the antiviral drug development strategies, and clinical drugs as well as new compounds are tested for their effect on it. Drugs like favipiravir, ribavirin, penciclovir, remdesivir, galidesivir, itraconazole, novobiocin, chenodeoxycholic acid, cortisone, idarubicin, silybin, pancuronium bromide, dabigatran etexilate 6 '-fluorinated-aristeromycin analogues, acyclovir, and fleximer analogues exhibited RdRp inhibition. ${ }^{7}$ In principle, selective inhibition of RdRp by these agents could not cause significant side effects and toxicity on host cells. ${ }^{8}$ In addition, natural compounds and their derivatives with anti-inflammatory, anti-tumor, and antivirus effects such as gnidicin and gniditrin from Gnidia lamprantha, and betulonal from Cassine xylocarpa showed high binding affinity to $\mathrm{RdRp}$ with promising anti-COVID-19 activity, though further investigations are needed. $^{9}$

\section{Papain-Like Protease (PLpro)}

PLpro exists in all coronaviruses and has been found to be vital for the release of non-structure proteins 1, 2, and 3 from the N-terminal part of polyproteins $1 \mathrm{a}$ and $1 \mathrm{ab} .{ }^{10}$ In addition, the PLpro of SARS-COV has been shown to have deubiquitinating and interferon antagonism activities, thus preventing interferon-regulatory factor 3 (IRF3) activation and blocking the nuclear factor-j-light-chain-enhancer of activated $B$ cells (NFjB) pathway. ${ }^{11}$ This enzyme is considered to be a potential antiviral drug target. Clinically known protease inhibitors such as disulfiram, lopinavir, and ritonavir have been reported to be active against Middle East respiratory syndrome (MERS) and severe acute respiratory syndrome coronavirus (SARS-COV). ${ }^{12,13}$ Despite the lack of clinical evidence, disulfiram has been revealed to inhibit MERS and SARS papain-like protease in cell cultures. On the other hand, clinical trials have been initiated to test ritonavir and lopinavir in patients infected with COVID-19. ${ }^{14}$ These drugs were initially assumed to inhibit SARS and MERS 3-chymotrypsin-like protease, and appeared to be associated with SARS patients' improved clinical outcomes in a nonrandomized open-label trial. Nevertheless, it is debatable whether these drugs could effectively inhibit COVID-19 papain-like and 3-chymotrypsin-like proteases. ${ }^{14}$ Besides, a study reported that 6-thioguanine (6TG) and 6-mercaptopurine (6MP) inhibit SARS-COV PLpro. ${ }^{15}$

\section{Helicases}

Helicases are universal motor proteins that separate and/or rearrange duplexes of nucleic acid in reactions driven by hydrolysis of adenosine triphosphate (ATP). ${ }^{16}$ Nsp13, a superfamily 1 helicase, is a multi-functional protein with an N-terminal metal binding domain (MBD) and a helicase domain (Hel). The N-terminal forms a $\mathrm{Zn}$ binding domain while the $\mathrm{C}$-terminal forms a helicase domain with a conserved motif, and participate in unravelling double-stranded (ds) DNA and RNA of the virus along the $5^{\prime}-3^{\prime}$ direction in a nucleoside triphosphate-dependent manner. A study reports that Nsp13 dependent unravelling is an essential process for the replication, transcription, and translation of SARS-COV-2. ${ }^{17}$ Therefore, helicases are promising anti-COVID-19 drug targets. Different scientific literature has recently reported various potent inhibitors of helicases encoded by SARS-COV-2. Some inhibitors like bananins, 5-hydroxychromone derivative, ADKs, and SSYA10-001 are under preclinical studies for the treatment of SARS-COV-2. ${ }^{18}$

\section{3-Chymotrypsin-Like Protease (3CLpro)}

3CLpro (Nsp5) mediates Nsps maturation, which is vital for the life cycle of the virus. 3CLpro is first cleaved from poly-proteins to produce mature enzymes, and then further cleaves downstream Nsps at 11 sites to release Nsp4Nsp16. ${ }^{19}$ In-depth investigation of the structure and catalytic mechanism of 3CLpro makes 3CLpro an interesting target for anti-COVID-19 drug development. Peptide inhibitors and small-molecule inhibitors are targeting 3CLpro of SARS-COV. ${ }^{20}$ Velpatasvir, ledipasvir, lymecycline, demeclocycline, doxycycline, oxytetracycline, nicardipine, 
telmisartan, conivaptan, and montelukast exhibited highest binding affinity to 3 CLpro. ${ }^{20}$ In silico studies revealed that bepotastine, colistin, epirubicin, valrubicin, icatibant, epoprostenol, vapreotide, caspofungin, aprepitant, and perphenazine also bind to the lopinavir/ritonavir-binding site on SARS-COV. ${ }^{21}$

\section{The Viral Structural Proteins Spike Protein}

The genome of beta-coronavirus encodes many structural proteins, including the glycosylated spike $(\mathrm{S})$ protein which is a major inducer of host immune responses. ${ }^{22}$ The spike protein is a clove-shaped type I-transmembrane protein. This protein has three segments. These are: the ectodomain (ED) region, transmembrane region, and intracellular domain. The intracellular domain comprises the intracellular short tail part, the receptor binding S1 domain (three S1 heads), and the membrane fusion subunit S2 (trimeric stalk) on the C-terminal together with the ED. ${ }^{23,24}$ Membrane fusion and virus entry activation require the cleavage at the junction of S1-S2. Hence, due to its vital role in the interaction between the virus and the cell receptor, spike protein is an important potential target for antiviral agents. ${ }^{25}$

Consequently, monoclonal antibodies targeting the S1 subunit and fusion inhibitors targeting the S2 subunit may be effective therapeutic agents to treat COVID-19 infections. A serine endoprotease, furin, cleaves off $\mathrm{S} 1-\mathrm{S} 2$ and, thus, could be a suitable anti-COVID-19 agent. $^{26}$ A red-algaderived lectin, griffithsin, binds to SARS-COV spike glycoprotein and HIV glycoprotein $120 .^{27}$ As a result, griffithsin has been tested for HIV prevention in Phase I studies as a gel or an enema and produced promising effect. However, the delivery systems and potency of $\mathrm{S}$ inhibitors in general should be re-evaluated for the prevention or treatment of COVID-19. ${ }^{27}$ In addition, nafamostat which is under clinical investigation for COVID-19 treatment inhibits spikemediated membrane fusion of the virus. Moreover, Yang et al revealed that, in a mouse model, a DNA vaccine encoding the full-length $\mathrm{S}$ protein SARS-COV urbani strain could induce both the responses of protective immunity and production of neutralizing antibody by the T cells. ${ }^{28}$

\section{Envelope, Nucleocapsid, and Membrane Proteins}

The E (envelope) protein is the smallest transmembrane structural protein of coronaviruses. ${ }^{29}$ Two different domains constitute the E protein: the charged cytoplasmic tail and the hydrophobic domain. The E protein possesses vital biological functions for the structural integrity coronaviruses and host virulence. ${ }^{30}$ The other structural protein, nucleocapsid (N) protein, is conserved across different members of the coronavirus families. ${ }^{31}$ The most important function of the $\mathrm{N}$ protein $\mathrm{N}$-terminal domain (NTD) is binding of RNA, while the primary function of the C-terminal domain (CTD) is dimerization. ${ }^{31,32}$ On the other hand, the main function of the membrane (M) protein is maintenance of the shape of the viral envelope. It performs this activity by interacting with other coronavirus proteins, incorporating Golgi complex into new virions, and stabilizing of $\mathrm{N}$ protein. ${ }^{33}$

Therefore, E, N, and $\mathrm{M}$ proteins can be considered as targets for the development of anti-COVID-19 drugs. Many antiviral, anti-bacterial, anti-asthmatic, anti-inflammatory. and anti-tumor drugs were found to have comparatively good affinity to these targets, thereby inhibiting the viral replication in the host cells. Moreover, small interfering RNAs (siRNAs) showed strong binding affinity for 220-241 and 460-480 regions of M protein mRNA. ${ }^{9}$

\section{The Host-Specific Receptor or Enzymes}

Based on the result of many findings, the host ACE2 has been evidenced to be the specific receptor for the spike RNA binding domain (RBD) of SARS-COV. ${ }^{34}$ A recent study shows that the host receptor of SARS-COV-2 is similar to SARS-COV. In addition, the spike RBD sequence of SARS-COV-2 is also found to be nearly identical to SARS-COV RBD. Moreover, an interaction between the key amino acid residue of $\mathrm{RBD}$ receptorbinding motif and ACE2 is an important process for antiCOVID-19 drug development. ${ }^{34}$ According to the present research progress, ACE2 is considered as a host target for the discovery of COVID-19 therapy. Clinical drugs such as troglitazone, losartan, ergotamine, cefmenoxime, and silybin were found to bind with ACE2 receptor with low energy. $^{35}$

On the other hand, the enzyme transmembrane protease, serine 2 (TMPRSS2), triggers the infection of SARS-COV and MERS-COV by cutting the viral spike protein. $^{35}$ Inhibiting the enzymatic activity of TMPRSS2 can prevent some coronaviruses from entering into host cells. To this effect, pivampicillin, hetacillin, cefoperazone, clindamycin, kouitchenside I, phyllaemblicin G7, 
and neoandrographolide are predicted to be potential inhibitors of TMPRSS $2 .{ }^{36}$

\section{The Viral Virulence Factors}

A study showed that coronaviruses have three virulence factors which are Nsp1, Nsp3c, and ORF7a that interfere with the host's innate immunity and assist virus immune escape. ${ }^{37}$ Nsp1 interacts with the host 40 S ribosomal subunit that triggers mainly host mRNA degradation and also inhibits production of type-I interferon. ${ }^{37}$ On the other hand, Nsp3c has the ability to bind with the host's adenosine diphosphate (ADP)-ribose to help coronaviruses to resist host innate immunity. ${ }^{38}$ ORF7a directly binds to bone marrow matrix antigen 2 (BST-2) and inhibits its activity by blocking the glycosylation of BST-2. ${ }^{39}$ BST-2 is responsible for inhibition of the release of newlyassembled coronaviruses from host cells. These evidences suggest that Nsp1, Nsp3c, and ORF7a may be potential targets for anti-COVID-19 drug development. ${ }^{40}$ Studies showed that many of the clinical drugs and natural products with anti-bacterial and anti-inflammatory effects, such as piperacillin, cefpiramide, streptomycin, lymecycline, and tetracycline, exhibited relatively high binding affinity to these three target proteins. ${ }^{41}$

\section{Pro-Inflammatory Cytokines}

Cytokines are glycoproteins that play as chemical signals in the immune response to pathogen. Our immune cells produce so many cytokines in the body. Out of these, the pro-inflammatory cytokines have deleterious impact when they are released in response to viruses like SARS-COV-2. SARS-COV-2 infects the lower and upper respiratory tract and causes mild or highly acute respiratory syndrome with subsequent release of pro-inflammatory cytokines like interleukin (IL)-1 $\beta$ and IL-6. ${ }^{41}$ Its binding to the Toll-like receptor (TLR) in the lung commonly causes the release of pro-IL-1 $\beta$ which is cleaved by caspase-1, followed by inflammasome activation and production of active mature IL- $1 \beta$ that is a mediator of lung inflammation, fever, and fibrosis. ${ }^{42}$

Pro-inflammatory IL-1 family members and IL-6 suppression are expected to produce a therapeutic effect in COVID-19. This could be achieved by non-inflammatory cytokines such as IL-37 and IL-38. IL-37 has the capability to inhibit inflammation by binding to IL-18Ra receptor, and suppress molecules of class II histocompatibility complex, IL-1 $\beta$, IL-6, and tumor necrotic factor. ${ }^{43,44}$ IL-37 suppresses IL-1 $\beta$ in an inflammatory state induced by
COVID-19 to produce a novel therapeutic effect. Similarly, the newest cytokine of the IL-1 family members, IL-38, is also a suppressor cytokine which interferes with IL-1 $\beta$ and other pro-inflammatory IL-family members. Moreover, it inhibits inflammation in viral infections including that caused by COVID-19, to be considered as a potential therapeutic cytokine. ${ }^{45}$

Furthermore, the Janus kinase/signal transducers and activators of transcription (JAK/STAT) pathway is a key signaling mechanism for many types of cytokines, and essential to cellular response to exogenous signals in the immune system. JAK inhibitors exhibited a significant role in inhibiting and blocking cytokine release. ${ }^{46}$ Potent and selective JAK-STAT signaling inhibitors baricitinib, fedratinib, and ruxolitinib are reported to be effective against the impacts of the higher levels of cytokines (including interferon- $\gamma$ ) particularly observed in patients with COVID-19. ${ }^{47}$ Besides, inositol-requiring transmembrane kinase/endoribonuclease 1a and tylophorine-based compounds showed antiCOVID-19 activities in different studies. ${ }^{46,48}$

\section{Conclusion and Perspectives}

In the near future, vaccines, therapeutic antibodies, cytokines, and nucleic acid-based therapies targeting viral structural glycoproteins, papain-like protease, RNAdependent RNA polymerase (RdRp), 3-chymotrypsin-like protease, helicases, IL-1, IL-6, and JAK/STAT signaling pathways should be developed to prevent and treat COVID-19. Despite the current development and various recently reported drug repositioning studies, there is no as such potent and selective approved drug for COVID-19 treatment. Therefore, more investigations are clearly need to be done soon enough to get rid of the catastrophic impacts of COVID-19. Indeed, ongoing in silico and preclinical investigations on different compounds are being carried out by research companies. But, still rapid clinical trials on these compounds and further investigations of novel compounds are needed unequivocally. Finally, vaccine development by considering the above mentioned targets is an indispensable task.

\section{Abbreviations}

COVID-19, coronavirus disease-2019; 3CLpro, 3-chymotrypsin-like protease; E, envelope; JAK/STAT, Janus kinase/signal transducers and activators of transcription; $\mathrm{M}$, membrane protein; N, nucleocapsid protein; Nsp, non-structure protein; PLpro, papain-like protease; RdRp, RNA-dependence RNA 
polymerase; SARS-COV-2, severe acute respiratory syndrome coronavirus-2; $\mathrm{S}$, spike.

\section{Acknowledgments}

The authors wish to acknowledge Addis Ababa University and Woldia University for providing internet access during the search of the articles.

\section{Disclosure}

The authors declare no competing interests.

\section{References}

1. World Health Organization. Coronavirus disease 2019 (COVID-19) situation reports, 2020

2. Ledford H. Coronavirus breakthrough: dexamethasone is first drug shown to save lives. Nature. 2020;582(7813):469. doi:10.1038/ d41586-020-01824-5

3. Grein J, Ohmagari N, Shin D, et al. Compassionate Use of Remdesivir for Patients with Severe Covid-19. N Engl J Med. 2020;382(24):2327-2336. doi:10.1056/NEJMoa2007016

4. Subissi L, Imbe RI, Ferron F, et al. SARS-CoV ORF1b-encoded nonstructural proteins 12-16: replicative enzymes as antiviral targets. Antiviral Res. 2014;101:122-130. doi:10.1016/j.antiviral.2013.11.006

5. Imbert I, Guillemot JC, Bourhis JM, et al. A second, non-canonical RNA-dependent RNA polymerase in SARS coronavirus. EMBO J. 2006;25:4933-4942. doi:10.1038/sj.emboj.7601368

6. Kirchdoerfer RN, Ward AB. Structure of the SARS-CoV nsp12 polymerase bound to nsp7 and nsp8 co-factors. Nat. Commun 2019;10(1):2342. doi:10.1038/s41467-019-10280-3

7. Wang M, Cao R, Zhang L, et al. Remdesivir and chloroquine effectively inhibit the recently emerged novel coronavirus (2019-nCoV) in vitro. Cell Res. 2019;30:269-271. doi:10.1038/ s41422-020-0282-0

8. Chu CK, Gadthula S, Chen X, et al. Antiviral activity of nucleoside analogues against SARS-coronavirus (SARS-coV). Antivir Chem Chemother. 2006;17:285-289. doi:10.1177/095632020601700506

9. Wu C, Liu Y, Yang Y, et al. Analysis of therapeutic targets for SARS-CoV-2 and discovery of potential drugs by computational methods. Acta Pharm Sin B. 2020;10(5):766-788. doi:10.1016/j. apsb.2020.02.008

10. Chen X, Yang X, Zheng Y, Yang Y, Xing Y, Chen Z. SARS coronavirus papain-like protease inhibits the type I interferon signaling pathway through interaction with the STING-TRAF3-TBK1 complex. Protein Cell. 2014;5(5):369-381. doi:10.1007/s13238014-0026-3

11. Holger AL, Nasser F, Viktoria L. The Papain-Like Protease from the Severe Acute Respiratory Syndrome Coronavirus is a Deubiquitinating Enzyme. J Virol. 2005;70(24):5199-15208.

12. Lin MH, Moses DC, Hsieh CH. Disulfiram can inhibit MERS and SARS coronavirus papain-like proteases via different modes. Antiviral Res. 2018;150:155-163. doi:10.1016/j.antiviral.2017.12.015

13. Sheahan TP, Sims AC, Leist SR. Comparative therapeutic efficacy of remdesivir and combination lopinavir, ritonavir, and interferon beta against MERS-CoV. Nature Commun. 2020;11(1):222. doi:10.1038/ s41467-019-13940-6

14. Arabi YM, Alothman A, Balkhy HH. Treatment of Middle East Respiratory Syndrome with a combination of lopinavir-ritonavir and interferon-betalb (MIRACLE trial): study protocol for a randomized controlled trial. Trials. 2018;19(1):81. doi:10.1186/ s13063-017-2427-0
15. Cheng KW, Cheng SC, Chen WY. Thiopurine analogs and mycophenolic acid synergistically inhibit the papain-like protease of Middle East respiratory syndrome coronavirus. Antiviral Res. 2015;115:9-16. doi:10.1016/j.antiviral.2014.12.011

16. Kwong AD, Rao BG, Jeang KT. Viral and cellular RNA helicases as antiviral targets. Nat Rev Drug Discov. 2005;4(10):845-853. doi:10.1038/nrd1853

17. Frick DN, Lam AMI. Understanding helicases as a means of virus control. Curr Pharm Des. 2006;12(11):1315-1338. doi:10.2174/ 138161206776361147

18. Kim MK, Yu MS, Park HR, et al. 2,6-Bis-arylmethyloxy-5-hydroxychromones with antiviral activity against both hepatitis $\mathrm{C}$ virus (HCV) and SARS-associated coronavirus (SCV). Eur J Med Chem. 2011;46(11):5698-5704. doi:10.1016/j.ejmech.2011.09.005

19. Yang H, Xie W, Xue X, et al. Design of wide-spectrum inhibitors targeting coronavirus main proteases. PLoS Biol. 2005;3(10):e324. doi:10.1371/journal.pbio.0030324

20. Pillaiyar T, Manickam M, Namasivayam K, et al. An overview of severe acute respiratory syndrome-coronavirus (SARS-CoV) 3CL protease inhibitors: peptidomimetics and small molecule chemotherapy. $J$ Med Chem. 2016;59(14):6595-6628. doi:10.1021/acs.jmedchem.5b01461

21. Liu X, Wang XJ. Potential Inhibitors for 2019-nCoV coronavirus $\mathrm{m}$ protease from clinically approved medicines. Bio Rxiv. 2020;01:29.

22. Du L, He Y, Zhou Y, et al. The spike protein of SARS-CoV - A target for vaccine and therapeutic development. Nat. Rev. Microbiol. 2009;7 (3):226-236

23. Yuan Y, Cao D, Zhang Y, et al. Cryo-EM structures of MERS-CoV and SARS-CoV spike glycoproteins reveal the dynamic receptor binding domains. Nat Commun. 2017;8(1):15092. doi:10.1038/ncomms15092

24. Du L, He Y, Zhou Y, Liu S, Zheng B-J, Jiang S. The spike protein of SARS-CoV-2, a target for vaccine and therapeutic development. Nat Rev Microbiol. 2009;7(3):226-236. doi:10.1038/nrmicro2090

25. Wrapp D, Wang N, Corbett KS, et al. Cryo-EM structure of the 2019-nCoV Spike in the prefusion conformation. Science. 2020;367 (6483):1260-1263. doi:10.1126/science.abb2507

26. Millet JK, Whittaker GR. Host cell entry of Middle East respiratory syndrome coronavirus after two-step, furin-mediated activation of the spike protein. Proc Natl Acad Sci. 2014;11(42):15214-15219. doi:10.1073/pnas.1407087111

27. O'Keefe BR, Giomarelli B, Barnard DL. Broad-spectrum in vitro activity and in vivo efficacy of the antiviral protein griffithsin against emerging viruses of the family Coronaviridae. J Virol. 2010;84 (5):2511-2521. doi:10.1128/JVI.02322-09

28. Barton C, Kouokam JC, Lasnik AB, et al. Activity of and effect of subcutaneous treatment with the broad-spectrum antiviral lectin griffithsin in two laboratory rodent models. Antimicrob Agents Chemother. 2014;58(1):120-127. doi:10.1128/AAC.01407-13

29. Kuo L, Hurst KR, Masters PS. Exceptional flexibility in the sequence requirements for coronavirus small envelope protein function. $J$ Virol. 2007;81(5):2249-2262. doi:10.1128/JVI.01577-06

30. Venkatagopalan P, Daskalova SM, Lopez LA, et al. Coronavirus envelope (E) protein remains at the site of assembly. Virology. 2015;478:75-85. doi:10.1016/j.virol.2015.02.005

31. Chang CK, Lo SC, Wang YS, Hou M-H. Recent insights into the development of therapeutics against coronavirus diseases by targeting N protein. Drug Discov Today. 2016;21(4):562-572. doi:10.1016/j. drudis.2015.11.015

32. McBride R, Van ZM, Fielding BC. The coronavirus nucleocapsid is a multifunctional protein. Viruses. 2014;6(8):299-3018. doi:10.3390/ v6082991

33. Schoeman D, Fielding BC. Coronavirus envelope protein: current knowledge. Virol J. 2019;16(1):69. doi:10.1186/s12985-019-1182-0

34. Donoghue $\mathrm{M}$, Hsieh F, Baronas $\mathrm{E}$, et al. A novel angiotensin-converting enzyme related carboxypeptidase (ACE2) converts angiotensin I to angiotensin 1-9. Circ Res. 2000;87(5):426. doi:10.1161/01.RES.87.5.e1 
35. Imai Y, Kuba K, Rao S, et al. Angiotensin-converting enzyme 2 protects from severe acute lung failure. Nature. 2005;436 (7047):112-116. doi:10.1038/nature03712

36. Glowacka I, Bertram S, Muller MA, et al. Evidence that TMPRSS2 activates the severe acute respiratory syndrome coronavirus spike protein for membrane fusion and reduces viral control by the humoral immune response. J Virol. 2011;85(9):4122-4134. doi:10.1128/JVI.02232-10

37. Kamitani W, Narayanan K, Huang C, et al. Severe acute respiratory syndrome coronavirus nsp1 protein suppresses host gene expression by promoting host mRNA degradation. Proc Natl Acad Sci. 2006;103 (34):12885-12890. doi:10.1073/pnas.0603144103

38. Narayanan K, Huang C, Lokugamage K, et al. Severe acute respiratory syndrome coronavirus nsp1 suppresses host gene expression, including that of type I interferon, in infected cells. J Virol. 2008;82 (9):4471-4479. doi:10.1128/JVI.02472-07

39. Forni D, Cagliani R, Mozzi A, et al. Extensive positive selection drives the evolution of nonstructural proteins in lineage $\mathrm{C}$ betacoronaviruses. J Virol. 2016;90(7):3627-3639. doi:10.1128/JVI.02988-15

40. Taylor JK, Coleman CM, Postel S, et al. Severe acute respiratory syndrome coronavirus ORF7a inhibits bone marrow stromal antigen 2 virion tethering through a novel mechanism of glycosylation interference. J Virol. 2015;89(23):11820-11833. doi:10.1128/JVI.02274-15

41. Dinarello CA, Conti P, Mier JW. Effects of human interleukin-1 on natural killer cell activity: is fever a host defense mechanism for tumor killing. Yale J Biol Med. 1986;59(2):97-106.
42. Conti P, Gallenga CE, Tetè G. How to reduce the likelihood of coronavirus-19 (CoV-19 or SARS-CoV-2) infection and lung inflammation mediated by IL-1. J Biol Regul Homeost Agents. 2020;34(2):10.

43. Conti P, Lauritano D, Caraffa A. Microglia and mast cells generate proinflammatory cytokines in the brain and worsen inflammatory state: suppressor effect of IL-37. Eur J Pharmacol. 2020;875:173035. doi:10.1016/j.ejphar.2020.173035

44. Heoharides TC, Tsilioni I, Conti P. Mast cells may regulate the anti-inflammatory activity of IL-37. Int J Mol Sci. 2019;20:15.

45. Lauritano D, Ronconi G, Caraffa A, et al. New aspect of allergic contact dermatitis, an inflammatory skin disorder mediated by mast cells: can IL-38 help? Med Hypotheses. 2020;139:109687. doi:10.1016/j.mehy.2020.109687

46. Russell B, Moss C, George G, et al. Associations between immunesuppressive and stimulating drugs and novel COVID-19-a systematic review of current evidence. ecancer. 2020;14:1022. doi:10.3332/ ecancer.2020.1022

47. Kontzias A, Kotlyar A, Laurence A, Changelian P, O’Shea JJ. Jakinibs: a new class of kinase inhibitors in cancer and autoimmune disease. Curr Opin Pharmacol. 2012;12(4):464-470. doi:10.1016/j. coph.2012.06.008

48. Richardson P, Griffin I, Tucker C. Baricitinib as potential treatment for 2019-nCoV acute respiratory disease. Lancet. 2020;395 (10223):497-506. doi:10.1016/S0140-6736(20)30304-4

\section{Publish your work in this journal}

Biologics: Targets and Therapy is an international, peer-reviewed journal focusing on the patho-physiological rationale for and clinical application of Biologic agents in the management of autoimmune diseases, cancers or other pathologies where a molecular target can be identified. This journal is indexed on PubMed Central, CAS, EMBase,
Scopus and the Elsevier Bibliographic databases. The manuscript management system is completely online and includes a very quick and fair peer-review system, which is all easy to use. Visit http://www.dovepress.com/testimonials.php to read real quotes from published authors. 